\title{
Weak Conservation Laws for Minimizers which are not Pontryagin Extremals*
}

\author{
Delfim F. M. Torres \\ delfim@mat.ua.pt \\ Department of Mathematics \\ University of Aveiro \\ 3810-193 Aveiro, Portugal
}

\begin{abstract}
We prove a Noether-type symmetry theorem for invariant optimal control problems with unrestricted controls. The result establishes weak conservation laws along all the minimizers of the problems, including those minimizers which do not satisfy the Pontryagin Maximum Principle.
\end{abstract}

Mathematics Subject Classification 2000: 49K15, 70H33, 37J15.

Keywords. Noether's symmetry theorem, invariant optimal control problems, gap between optimality and existence, weak conservation laws.

\section{Introduction}

Emmy Noether's first theorem [11] is one of the most beautiful and fundamental results of the calculus of variations. The result comprises a universal principle, connecting the existence of a family of transformations under which the functional to be minimized is invariant (the existence of variational symmetries) with the existence of conservation laws (first integrals of the Euler-Lagrange differential equations). Conservation laws can then be used to simplify the problem of finding the minimizers. They have played an important role, both in mathematics and physics, since the birth of the calculus of variations in the eighteen century, having been extensively used by giants like the Bernoulli brothers, Newton, Leibniz, Euler, Lagrange, and Legendre. Conservation laws, obtained from Noether's theorem, have a profound effect on a vast number of disciplines, ranging from classical mechanics, where they find important interpretations such as

${ }^{*}$ Research report CM05/I-11. Accepted for presentation (Paper No: 113) at the 2nd International Conference "Physics and Control" (PhysCon 2005), August 24-26, 2005, Saint Petersburg, Russia. To appear in the respective Conference Proceedings. 
conservation of energy, conservation of momentum, or conservation of angular momentum, to engineering, economics, control theory and their applications [8].

The first extension of Noether's theorem to the more general context of optimal control was published in 1973 [5]. Since then, many Noether-like theorems have been obtained in the context of optimal control - see [17 and references therein. We recall that all such versions of Noether's theorem assume the Pontryagin maximum principle [13] to be satisfied, and use its conditions, including the adjoint system, in their proofs.

Optimal control with unbounded controls is an area of strong current activity, because of numerous applications involving modern technology such as "smart materials" 9. When there are no restrictions on the values of the control variables, as in the calculus of variations, it is well known that optimal control problems may present solutions for which the Pontryagin Maximum Principle fails to be satisfied (see e.g. [19, §11.1]). This is due to the fact that the hypotheses of the existence theory need to be complemented with additional regularity conditions in order to proceed with the arguments which lead to the maximum principle [15]: unboundedness of the controls "propagates" through the dynamical control system, often causing a lack of regularity for the solutions.

In spite of the gap between the hypotheses of necessary optimality conditions and existence theorems, J. Ball proved [1] that, for time-invariant problems, the conservation of the Hamiltonian (conservation of energy) is still valid for minimizers which might not satisfy the Euler-Lagrange necessary condition. More recently, in 2002, G. Francfort and J. Sivaloganathan proposed a generalization of Ball's result, giving some applications to hyper-elasticity [7].

In this note we extend the previous results [7] from the calculus of variations framework to the optimal control setting. We obtain weak conservation laws for minimizers which do not necessarily satisfy the Pontryagin maximum principle.

\section{Bad Behavior in Optimal Control}

The optimal control problem consists to minimize a cost functional

$$
I[x(\cdot), u(\cdot)]=\int_{a}^{b} L(t, x(t), u(t)) d t
$$

subject to a control system described by ordinary differential equations

$$
\dot{x}(t)=\varphi(t, x(t), u(t))
$$

together with certain appropriate endpoint conditions. The Lagrangian $L$ : $[a, b] \times \mathbb{R}^{n} \times \mathbb{R}^{m} \rightarrow \mathbb{R}$ and the velocity vector $\varphi:[a, b] \times \mathbb{R}^{n} \times \mathbb{R}^{m} \rightarrow \mathbb{R}^{n}$ are given, and assumed to be smooth: $L(\cdot, \cdot, \cdot), \varphi(\cdot, \cdot, \cdot) \in C^{1}$. We are interested in the case where there are no restrictions on the control set: $u(t) \in \mathbb{R}^{m}$. We denote the problem by $(P)$. In the particular case $\varphi(t, x, u)=u$, one obtains the fundamental problem of the calculus of variations, which covers all classical mechanics. The choice of the classes $\mathcal{X}$ and $\mathcal{U}$, respectively of the state 
$x:[a, b] \rightarrow \mathbb{R}^{n}$ and control variables $u:[a, b] \rightarrow \mathbb{R}^{m}$, play an important role in our discussion. In connection with the Pontryagin maximum principle, the optimal controls are typically assumed to be essentially bounded, $\mathcal{U}=L_{\infty}$ [13; while to guarantee existence, compactness arguments require a bigger class of measurable control functions, $\mathcal{U}=L_{1}[3]$. Given an optimal control problem with unrestricted controls, it may happen that the Pontryagin maximum principle is valid, while existence of minimizers is not guaranteed; or it may happen that the minimizers predicted by the existence theory fail to be Pontryagin extremals. Conservation laws are obtained from Noether's theorem, assuming that the minimizers are Pontryagin extremals. In this work our objective is to prove weak conservation laws valid for bad-behaved problems with minimizers which are not Pontryagin extremals. We begin to explain why bad-behavior can occur.

The Pontryagin maximum principle is a necessary optimality condition which can be obtained from a general Lagrange multiplier theorem in spaces of infinite dimension (cf. e.g. 10 12]). Introducing the Hamiltonian function

$$
H(t, x, u, \psi)=-L(t, x, u)+\psi \cdot \varphi(t, x, u),
$$

where $\psi_{i}, i=1, \ldots, n$, are the "Lagrange multipliers" or the "generalized momenta", the multiplier theorem asserts that the optimal control problem is equivalent to the maximization of the augmented functional

$$
J[x(\cdot), u(\cdot), \psi(\cdot)]=\int_{a}^{b}(H(t, x(t), u(t), \psi(t))-\psi(t) \cdot \dot{x}(t)) d t .
$$

Let us assume, for simplicity, $\mathcal{X}=C^{1}\left([a, b] ; \mathbb{R}^{n}\right), \mathcal{U}=C\left([a, b] ; \mathbb{R}^{m}\right)$. Let $(\tilde{x}(\cdot), \tilde{u}(\cdot), \tilde{\psi}(\cdot))$ solve the problem, and consider arbitrary $C^{1}$-functions $h_{1}, h_{3}$ : $[a, b] \rightarrow \mathbb{R}^{n}, h_{1}(\cdot)$ vanishing at $a$ and $b\left(h_{1}(\cdot) \in C_{0}^{1}([a, b])\right)$, and arbitrary continuous $h_{2}:[a, b] \rightarrow \mathbb{R}^{m}$. Let $\varepsilon$ be a scalar. By definition of maximizer, we have

$$
J\left[\left(\tilde{x}+\varepsilon h_{1}\right)(\cdot),\left(\tilde{u}+\varepsilon h_{2}\right)(\cdot),\left(\tilde{\psi}+\varepsilon h_{3}\right)(\cdot)\right] \leq J[\tilde{x}(\cdot), \tilde{u}(\cdot), \tilde{\psi}(\cdot)],
$$

and one has the following necessary condition:

$$
\left.\frac{d}{d \varepsilon} J\left[\left(\tilde{x}+\varepsilon h_{1}\right)(\cdot),\left(\tilde{u}+\varepsilon h_{2}\right)(\cdot),\left(\tilde{\psi}+\varepsilon h_{3}\right)(\cdot)\right]\right|_{\varepsilon=0}=0 .
$$

Differentiating (4) gives

$$
\begin{aligned}
0=\int_{a}^{b} & \frac{\partial H}{\partial x}(t, \tilde{x}(t), \tilde{u}(t), \tilde{\psi}(t)) \cdot h_{1}(t)+\frac{\partial H}{\partial u}(t, \tilde{x}(t), \tilde{u}(t), \tilde{\psi}(t)) \cdot h_{2}(t) \\
& \left.+\frac{\partial H}{\partial \psi}(t, \tilde{x}(t), \tilde{u}(t), \tilde{\psi}(t)) \cdot h_{3}(t)-h_{3}(t) \cdot \dot{\tilde{x}}(t)-\tilde{\psi}(t) \cdot \dot{h}_{1}(t)\right] d t .
\end{aligned}
$$


Integrating the $\tilde{\psi}(t) \cdot \dot{h}_{1}(t)$ term by parts, and having in mind that $h_{1}(a)=$ $h_{1}(b)=0$, one derives

$$
\begin{array}{r}
\int_{a}^{b}\left[\left(\frac{\partial H}{\partial x}(t, \tilde{x}(t), \tilde{u}(t), \tilde{\psi}(t))+\dot{\tilde{\psi}}(t)\right) \cdot h_{1}(t)+\frac{\partial H}{\partial u}(t, \tilde{x}(t), \tilde{u}(t), \tilde{\psi}(t)) \cdot h_{2}(t)\right. \\
\left.+\left(\frac{\partial H}{\partial \psi}(t, \tilde{x}(t), \tilde{u}(t), \tilde{\psi}(t))-\dot{\tilde{x}}(t)\right) \cdot h_{3}(t)\right] d t=0
\end{array}
$$

Note that (6) was obtained for any variation $h_{1}(\cdot), h_{2}(\cdot)$, and $h_{3}(\cdot)$. Choosing $h_{1}(t)=h_{2}(t) \equiv 0$, and $h_{3}(\cdot)$ arbitrary, one obtains the control system (11):

$$
\dot{\tilde{x}}(t)=\frac{\partial H}{\partial \psi}(t, \tilde{x}(t), \tilde{u}(t), \tilde{\psi}(t)), \quad t \in[a, b] .
$$

With $h_{1}(\cdot)$ arbitrary, and $h_{2}(t)=h_{3}(t) \equiv 0$, we obtain the adjoint system:

$$
\dot{\tilde{\psi}}(t)=-\frac{\partial H}{\partial x}(t, \tilde{x}(t), \tilde{u}(t), \tilde{\psi}(t)), \quad t \in[a, b] .
$$

Finally, with $h_{2}(\cdot)$ arbitrary, and $h_{1}(t)=h_{3}(t) \equiv 0$, the maximality condition is obtained:

$$
\frac{\partial H}{\partial u}(t, \tilde{x}(t), \tilde{u}(t), \tilde{\psi}(t))=0, \quad t \in[a, b] .
$$

A necessary optimality condition for $(\tilde{x}(\cdot), \tilde{u}(\cdot))$ to be a minimizer of problem $(P)$ is given by the Pontryagin maximum principle: there exists $\tilde{\psi}(\cdot)$ such that the 3 -tuple $(\tilde{x}(\cdot), \tilde{u}(\cdot), \tilde{\psi}(\cdot))$ satisfy all the conditions (7), (8), and (9). We recall that conditions (7), (8), and (9) imply the equality

$$
\frac{d}{d t} H(t, \tilde{x}(t), \tilde{u}(t), \tilde{\psi}(t))=\frac{\partial H}{\partial t}(t, \tilde{x}(t), \tilde{u}(t), \tilde{\psi}(t)) .
$$

For piecewise smooth state trajectories, $\mathcal{X}=P C^{1}\left([a, b] ; \mathbb{R}^{n}\right)$, and piecewise continuous controls, $\mathcal{U}=P C\left([a, b] ; \mathbb{R}^{m}\right)$, similar arguments than those used to derive conditions (7), (8), and (9) continue to be justifiable. In fact, as already mentioned, the arguments can be carried out for essentially bounded controls, $\mathcal{U}=L_{\infty}\left([a, b] ; \mathbb{R}^{m}\right)$, and Lipschitzian state variables, $\mathcal{X}=W_{1, \infty}\left([a, b] ; \mathbb{R}^{n}\right)$. But if one took $\mathcal{U}=L_{1}\left([a, b] ; \mathbb{R}^{m}\right)$, and $\mathcal{X}=W_{1,1}\left([a, b] ; \mathbb{R}^{n}\right)$, as required by the existence theory, integration by parts of $\tilde{\psi}(t) \cdot \dot{h}_{1}(t)$ in (5) can no longer be justified, and one can not conclude with the adjoint system (8) and equality (10). This is more than a technical difficulty, and explains the possibility of badbehavior illustrated by the Ball-Mizel example [2. In the Ball-Mizel problem one has $n=m=1, L(t, x, u)=\left(x^{3}-t^{2}\right)^{2} u^{14}+\epsilon u^{2}$, and $\varphi(t, x, u)=u$ :

$$
\begin{gathered}
\int_{0}^{1}\left[\left(x^{3}(t)-t^{2}\right)^{2} u(t)^{14}+\epsilon u(t)^{2}\right] d t \longrightarrow \min , \\
\dot{x}(t)=u(t), \\
x(0)=0, \quad x(1)=k .
\end{gathered}
$$


For some values of the constants $\epsilon$ and $k$ there exists the unique optimal control $\tilde{u}(t)=\frac{2 k}{3} t^{-1 / 3}$ [4, which belongs to $L_{1}$ but not to $L_{\infty}$. The Pontryagin maximum principle is not satisfied since the adjoint system (8)

$$
\dot{\tilde{\psi}}(t)=-\frac{\partial H}{\partial x}(t, \tilde{x}(t), \tilde{u}(t), \tilde{\psi}(t)) \cong t^{-4 / 3}
$$

is not integrable. In this paper we obtain a new version of Noether's theorem, without using the adjoint system (8) and the property (10). This makes Noether's principle valid both for well- and bad-behaved optimal control problems.

\section{Conservation Laws in Optimal Control}

In 1918 Emmy Noether established the key result to find conservation laws in the calculus of variations 11, 18. We sketch here the standard argument used to derive Noether's theorem and conservation laws in the optimal control setting (cf. e.g. [5, 14]).

Let us consider a one-parameter group of $C^{1}$-transformations of the form

$h^{s}(t, x, u, \psi)=\left(h_{t}(t, x, u, \psi, s), h_{x}(t, x, u, \psi, s), h_{u}(t, x, u, \psi, s), h_{\psi}(t, x, u, \psi, s)\right)$,

where $s$ denote the independent parameter of the transformations. We require that to the parameter value $s=0$ there corresponds the identity transformation:

$$
\begin{aligned}
h^{0}(t, x, u, \psi) & =\left(h_{t}(t, x, u, \psi, 0), h_{x}(t, x, u, \psi, 0), h_{u}(t, x, u, \psi, 0), h_{\psi}(t, x, u, \psi, 0)\right) \\
& =(t, x, u, \psi)
\end{aligned}
$$

Associated to the group of transformations (11) we consider the infinitesimal generators

$$
\begin{gathered}
T(t, x, u, \psi)=\left.\frac{d}{d s} h_{t}(t, x, u, \psi, s)\right|_{s=0}, \quad X(t, x, u, \psi)=\left.\frac{d}{d s} h_{x}(t, x, u, \psi, s)\right|_{s=0}, \\
U(t, x, u, \psi)=\left.\frac{d}{d s} h_{u}(t, x, u, \psi, s)\right|_{s=0}, \quad \Psi(t, x, u, \psi)=\left.\frac{d}{d s} h_{\psi}(t, x, u, \psi, s)\right|_{s=0} .
\end{gathered}
$$

Definition 1. The optimal control problem $(P)$ is said to be invariant under a one-parameter group of $C^{1}$-transformations (11) if, and only if,

$$
\begin{aligned}
& \frac{d}{d s}\left\{\left[H\left(h^{s}(t, x(t), u(t), \psi(t))\right)\right.\right. \\
- & \left.\left.h_{\psi}(t, x(t), u(t), \psi(t), s) \cdot \frac{\frac{d h_{x}(t, x(t), u(t), \psi(t), s)}{d t}}{\frac{d h_{t}(t, x(t), u(t), \psi(t), s)}{d t}}\right] \frac{d h_{t}(t, x(t), u(t), \psi(t), s)}{d t}\right\}\left.\right|_{s=0}=0,
\end{aligned}
$$


with $H$ the Hamiltonian (2).

Having in mind (12), condition (14) is equivalent to

$$
\frac{\partial H}{\partial t} T+\frac{\partial H}{\partial x} \cdot X+\frac{\partial H}{\partial u} \cdot U+\frac{\partial H}{\partial \psi} \cdot \Psi-\Psi \cdot \dot{x}(t)-\psi(t) \cdot \frac{d}{d t} X+H \frac{d}{d t} T=0,
$$

where here, and to the end of the paper, all functions are evaluated at $(t, x(t), u(t), \psi(t))$ whenever not indicated. Along a Pontryagin extremal $(x(\cdot), u(\cdot), \psi(\cdot))$ equalities (17), (8), (9), and (10) are in force, and (15) reduces to

$$
\frac{d H}{d t} T-\dot{\psi}(t) \cdot X-\psi(t) \cdot \frac{d X}{d t}+H \frac{d T}{d t}=0 \Leftrightarrow \frac{d}{d t}(\psi(t) \cdot X-H T)=0 .
$$

We have just proved Noether's theorem for optimal control problems.

Theorem 1 (Noether's Theorem). If the optimal control problem is invariant under (11), in the sense of Definition 1, then

$$
\psi(t) \cdot X(t, x(t), u(t), \psi(t))-H(t, x(t), u(t), \psi(t)) T(t, x(t), u(t), \psi(t))=c
$$

(c a constant; $t \in[a, b] ; T$ and $X$ are given according to (13); $H$ is the Hamiltonian (2)) is a conservation law, that is, (16) is valid along all the minimizers $(x(\cdot), u(\cdot))$ of $(P)$ which are Pontryagin extremals.

All available versions of Noether's theorem found in the literature are valid only for well-behaved optimal control problems (conservation laws are, by definition, valid for minimizers which are Pontryagin extremals). In the next section we provide the first optimal control version of Noether's theorem valid in presence of bad behavior (valid also for minimizers which are not Pontryagin extremals). For that we need a new notion of conservation law.

\section{Weak Conservation Laws in Optimal Control}

In 1879 Paul duBois-Reymond proved an important basic result. From duBoisReymond lemma we know that

$$
\int_{a}^{b}(\psi(t) \cdot X-H T) \dot{\theta}(t) d t=0, \quad \forall \theta(\cdot) \in C_{0}^{1}([a, b]),
$$

is a weak form of conservation law (16). Follows the main result of the paper.

Theorem 2. If the optimal control problem is invariant under (11), in the sense of Definition 1 then

$$
\begin{aligned}
& \int_{a}^{b}[\psi(t) \cdot X(t, x(t), u(t), \psi(t)) \\
&-H(t, x(t), u(t), \psi(t)) T(t, x(t), u(t), \psi(t))] \dot{\theta}(t) d t=0
\end{aligned}
$$


$\left(\theta(\cdot)\right.$ is an arbitrary $W_{1,1}([a, b] ; \mathbb{R})$ function satisfying $\left.\theta(a)=\theta(b)=0\right)$ holds along all the minimizers $(x(\cdot), u(\cdot), \psi(\cdot)) \in W_{1,1} \times L_{1} \times W_{1,1}$ of (3).

Proof. Replacing the parameter $s$ of the group (11) by function $s \theta(t)$, the infinitesimal generators are then given by

$$
\begin{aligned}
& \left.\frac{d}{d s} h_{t}(t, x, u, \psi, s \theta(t))\right|_{s=0}=T(t, x, u, \psi) \theta(t), \\
& \left.\frac{d}{d s} h_{x}(t, x, u, \psi, s \theta(t))\right|_{s=0}=X(t, x, u, \psi) \theta(t), \\
& \left.\frac{d}{d s} h_{u}(t, x, u, \psi, s \theta(t))\right|_{s=0}=U(t, x, u, \psi) \theta(t), \\
& \left.\frac{d}{d s} h_{\psi}(t, x, u, \psi, s \theta(t))\right|_{s=0}=\Psi(t, x, u, \psi) \theta(t),
\end{aligned}
$$

with $T, X, U$, and $\Psi$ as in (13), and the necessary and sufficient condition of invariance (15) takes the form

$$
\begin{array}{r}
\left(\frac{\partial H}{\partial t} T+\frac{\partial H}{\partial x} \cdot X+\frac{\partial H}{\partial u} \cdot U+\frac{\partial H}{\partial \psi} \cdot \Psi-\Psi \cdot \dot{x}(t)+H \frac{d T}{d t}-\psi(t) \cdot \frac{d X}{d t}\right) \theta(t) \\
+(H T-\psi(t) \cdot X) \dot{\theta}(t)=0
\end{array}
$$

Condition (5) with $h_{1}(t)=X \theta(t), h_{2}(t)=U \theta(t)$, and $h_{3}(t)=\Psi \theta(t)$, gives

$$
\begin{aligned}
\int_{a}^{b}\left[\left(\frac{\partial H}{\partial x} \cdot X+\frac{\partial H}{\partial u} \cdot U+\frac{\partial H}{\partial \psi} \cdot \Psi-\Psi \cdot \dot{x}(t)\right.\right. & \left.-\psi(t) \cdot \frac{d X}{d t}\right) \theta(t) \\
& -(\psi(t) \cdot X) \dot{\theta}(t)] d t=0 .
\end{aligned}
$$

Using (15) in (18) permits to write

$$
\int_{a}^{b}\left[\left(-\frac{\partial H}{\partial t} T-H \frac{d T}{d t}\right) \theta(t)-(\psi(t) \cdot X) \dot{\theta}(t)\right] d t=0 ;
$$

while, on the other hand, using (17) in (18), one obtains

$$
\int_{a}^{b}\left[\left(\frac{\partial H}{\partial t} T+H \frac{d T}{d t}\right) \theta(t)+(H T) \dot{\theta}(t)\right] d t=0 .
$$

The conclusion follows summing up (19) and (20):

$$
\int_{a}^{b}(H T-\psi(t) \cdot X) \dot{\theta}(t) d t=0 .
$$


Invariance under an infinite continuous group of transformations, which rather than dependence on parameters depend upon arbitrary functions, is considered by Noether in the original paper [11. This is sometimes called "the second Noether theorem". We refer the reader to 16 for an extension of the second Noether theorem to optimal control problems which are semi-invariant under symmetries depending upon $k$ arbitrary functions of the independent variable and their derivatives. Theorem 2 is easily formulated under more general notions of invariance.

Theorem 2] gives, from the invariance properties of the optimal control problems, weak conservation laws along all the minimizers, including those minimizers which does not satisfy the standard necessary optimality conditions like the Pontryagin Maximum Principle or the Euler-Lagrange differential equations. Such fact may be useful to identify more general classes of well- and bad-behaved problems in the calculus of variations and optimal control, e.g., to synthesize a broad class of invariant problems exhibiting the Lavrentiev phenomenon 6. This possibility is under investigation and will be addressed elsewhere.

\section{Acknowledgements}

This work was partially supported by the Portuguese Foundation for Science and Technology (FCT) through the Control Theory Group (cotg) of the Centre for Research in Optimization and Control (CEOC).

\section{References}

[1] J. M. Ball, Minimizers and the Euler-Lagrange equations, Trends and Applications of Pure Mathematics to Mechanics (Palaiseau, 1983), Lecture Notes in Phys., vol. 195, Springer, Berlin, 1984, pp. 1-4. [MR 755716 (86e:49022)] [Zbl 0547.73013]

[2] J. M. Ball and V. J. Mizel, One-dimensional variational problems whose minimizers do not satisfy the Euler-Lagrange equation, Arch. Rational Mech. Anal. 90 (1985), 325-388. [MR 801585 (86k:49002)] [Zbl 0585.49002]

[3] Lamberto Cesari, Optimization-theory and applications, Applications of Mathematics (New York), vol. 17, Springer-Verlag, New York, 1983, ISBN 0-387-90676-2, Problems with ordinary differential equations. [MR 688142 (85c:49001)] [Zbl 0506.49001]

[4] Frank H. Clarke and R. B. Vinter, On the conditions under which the Euler equation or the maximum principle hold, Appl. Math. Optim. 12 (1984), 73-79. [MR 756513 (85m:49051)] [Zbl 0559.49012]

[5] Đorđe S. Đukić, Noether's theorem for optimum control systems, Internat. J. Control (1) 18 (1973), 667-672. [MR 0341229 (49 \#5979)] [Zbl 0281.49009]

[6] M. Foss, Examples of the Lavrentiev phenomenon with continuous Sobolev exponent dependence, J. Convex Anal. 10 (2003), 445-464. [MR 2043868 (2004m:49044)] [Zbl pre02066990]

[7] G. Francfort and J. Sivaloganathan, On conservation laws and necessary conditions in the calculus of variations, Proc. Roy. Soc. Edinburgh Sect. A 132 (2002), 1361-1371. [MR 1950811 (2003j:49035)] [Zbl 1027.49015] 
[8] A. M. Gugushvili, O. G. Khutsishvili, V. K. Sesadze, G. N. Dalakishvili, N. A. Mchedlishvili, T. G. Khutsishvili, V. M. Kekenadze, and D. F. M. Torres, Symmetries and conservation laws in optimal control systems, Georgian Technical University, Tbilisi, 2003, ISBN 99940-14-53-6.

[9] Irena Lasiecka, Optimal control problems and Riccati equations for systems with unbounded controls and partially analytic generators - applications to boundary and point control problems, Functional Analytic Methods for Evolution Equations, Lecture Notes in Math., vol. 1855, Springer, Berlin, 2004, pp. 313-369.

[10] Antonio Leitão, Cálculo variacional e controle ótimo, Publicações Matemáticas do IMPA. [IMPA Mathematical Publications], Instituto de Matemática Pura e Aplicada (IMPA), Rio de Janeiro, 2001, ISBN 85-244-0171-0, $23^{\circ}$ Colóquio Brasileiro de Matemática. [23rd Brazilian Mathematics Colloquium]. [MR 1846384] [Zbl pre01659603] (Portuguese)

[11] Emmy Noether, Invariant variation problems, Transport Theory Statist. Phys. 1 (1971), 186-207, Translated from the German (Nachr. Akad. Wiss. Göttingen Math.-Phys. Kl. II 1918, 235-257). [MR 0406752 (53 \#10538)] [Zbl 0292.49008]

[12] Pablo Pedregal, Introduction to optimization, Texts in Applied Mathematics, vol. 46, Springer-Verlag, New York, 2004, ISBN 0-387-40398-1. [MR 2014219 (2004j:90002)] [Zbl 1036.90002]

[13] L. S. Pontryagin, V. G. Boltyanskiı̌, R. V. Gamkrelidze, and E. F. Mishchenko, Selected works. Vol. 4, Classics of Soviet Mathematics, Gordon \& Breach Science Publishers, New York, 1986, ISBN 2-88124-134-4, The mathematical theory of optimal processes; Edited and with a preface by R. V. Gamkrelidze Translated from the Russian by K. N. Trirogoff Translation edited by L. W. Neustadt With a preface by L. W. Neustadt and K. N. Trirogoff Reprint of the 1962 English translation. [MR 898009 (90a:01108)] [Zbl 0882.01027]

[14] Delfim F. M. Torres, Conservation laws in optimal control, Dynamics, Bifurcations, and Control (Kloster Irsee, 2001), Lecture Notes in Control and Inform. Sci., vol. 273, Springer, Berlin, 2002, pp. 287-296. [MR 1901565 (2003c:49037)] [Zbl pre01819752]

[15] _ Lipschitzian regularity of the minimizing trajectories for nonlinear optimal control problems, Math. Control Signals Systems 16 (2003), 158-174. [MR 2006825 (2004i:49047)] [Zbl 1041.93023]

[16] - Gauge symmetries and Noether currents in optimal control, Appl. Math. ENotes 3 (2003), 49-57 (electronic). [MR 1980565 (2004g:49038)] [Zbl pre01925174]

[17] Quasi-invariant optimal control problems, Port. Math. (N.S.) 61 (2004), 97-114. [MR 2040245] [Zbl 1042.49015]

[18] Bruce van Brunt, The calculus of variations, Universitext, Springer-Verlag, New York, 2004, ISBN 0-387-40247-0. [MR 2004181 (2004m:49001)] [Zbl 1039.49001]

[19] Richard Vinter, Optimal control, Systems \& Control: Foundations \& Applications, Birkhäuser Boston Inc., Boston, MA, 2000, ISBN 0-8176-4075-4. [MR 1756410 (2001c:49001)] [Zbl 0952.49001] 